\title{
Giant aneurysm of an aortocoronary venous bypass graft treated by an endovascular approach
}

\author{
Michał Sojkaํㄹ Anna Drelich-Zbroja ${ }^{1}$, Maryla Kuczyńska ${ }^{1}$, Elżbieta Czekajska-Chehab², Andrzej Tomaszewski ${ }^{3}$, \\ Tomasz Jargiełło ${ }^{1}$
}

${ }^{1}$ Department of Interventional Radiology and Neuroradiology, Medical University of Lublin, Lublin, Poland

2I Department of Medical Radiology, Medical University of Lublin, Lublin, Poland

${ }^{3}$ Department of Cardiology, Medical University of Lublin, Lublin, Poland

Adv Interv Cardiol 2019; 15, 4 (58): 492-494

DOI: https://doi.org/10.5114/aic.2019.90226

Aneurysmal transformation of a venous coronary bypass graft is a rare (incidence of $0.07 \%$ ), yet potentially fatal complication of coronary artery bypass grafting. It is postulated $>5$ years following coronary artery bypass graft (CABG) multiple factors contribute to the development of graft aneurysm, including atherosclerosis, endothelial dysfunction, changes in smooth muscle orientation in the proximity of valves [1] and trauma during surgical handling of the vein [2]. Establishing the final diagnosis is hampered by unspecific clinical presentation (chest pain, dyspnea), with nearly $1 / 3$ of cases being diagnosed incidentally [1]. In consequence, patients undergo extensive and time-consuming cardiological workup prior to treatment. Despite cardiac surgery remaining the mainstay of treatment for coronary bypass graft aneurysms, minimally invasive endovascular procedures constitute an accepted and effective alternative for patients with multiple comorbidities without mechanical complications [1-3].

We hereby present a unique case of a 71-year-old patient with a giant aneurysmal transformation of an SVG-OM graft resulting in worsening dyspnea due to pulmonary trunk compression, successfully treated by endovascular embolization.

A patient with an implantable cardioverter-defibrillator and a past history of multiple coronary arterial bypass grafting (Ao-DIAG-LAD, Ao-RCA, SVG-OM, LITALAD) and angioplasty of the Ao-DIAG-LAD graft was admitted due to worsening dyspnea. Coronary computed tomography (CT) angiography revealed the presence of a partially thrombosed SVG-OM bypass graft aneurysm, measuring $73 \times 66 \times 61 \mathrm{~mm}$ and causing pulmonary trunk narrowing to $11 \mathrm{~mm}$ in the anteroposterior (AP) view (Figure $1 \mathrm{~A}$ ); another fully thrombosed, smaller aneurysm was visible at the occluded distal segment of the graft. A third aneurysm was detected at the proximal Ao-DIAG-LAD graft; full patency of the previously stented graft with no filling of the aneurysm was observed. Although patients with mechanical complications of coronary graft aneurysms, e.g. compression of adjacent vascular structures, are routinely treated by classic cardiac surgery [1], it was decided to refer our patient for less invasive endovascular exclusion of the partially filling SVG-OM graft aneurysm due to extensive post-operative retrosternal fibrosis and signs of cardiac insufficiency (ejection fraction $(E F)=28 \%$ ). Based on distal graft impatency and severe compression symptoms, occlusion of the afferent graft segment was chosen as the best treatment option in order to promote aneurysm shrinkage. Deployment of a vascular plug seemed to be the method of choice due to a short ( $<15 \mathrm{~mm}$ ) landing zone, rapid, single-device vessel occlusion and no additional mass to be left within the aneurysm sac. Amplatzer Vascular Plug 4 (AVP4 - St. Jude Medical, MN, USA) $6 \times 11 \mathrm{~mm}$ was selected as the most appropriate device. Consecutive stages of the embolization procedure performed under local anesthesia are presented in Figures $1 \mathrm{~B}$ and C. Control angiography confirmed proper positioning of the occluder and lack of contrast filling in the bypass graft and aneurysm sac.

Coronary CT angiograms obtained at 3 and 12 months (Figure $1 \mathrm{D}$ ) follow-up confirmed effective occlusion of the bypass graft with complete thrombosis of the aneurysm sac. Aneurysm sac shrinkage to $67 \times 63 \times 58 \mathrm{~mm}$ and $60 \times 62 \times 51 \mathrm{~mm}$ was observed at 3 and 12 months follow-up, respectively. This was accompanied by pulmo-

\section{Corresponding author:}

Dr. Maryla Kuczyńska, Department of Interventional Radiology and Neuroradiology, Medical University of Lublin, 8 Jaczewskiego St, 20-954 Lublin, Poland, phone: +48 607141 921, e-mail: maryla.kuczynska@gmail.com

Received: 9.01.2019, accepted; 12.08.2019. 

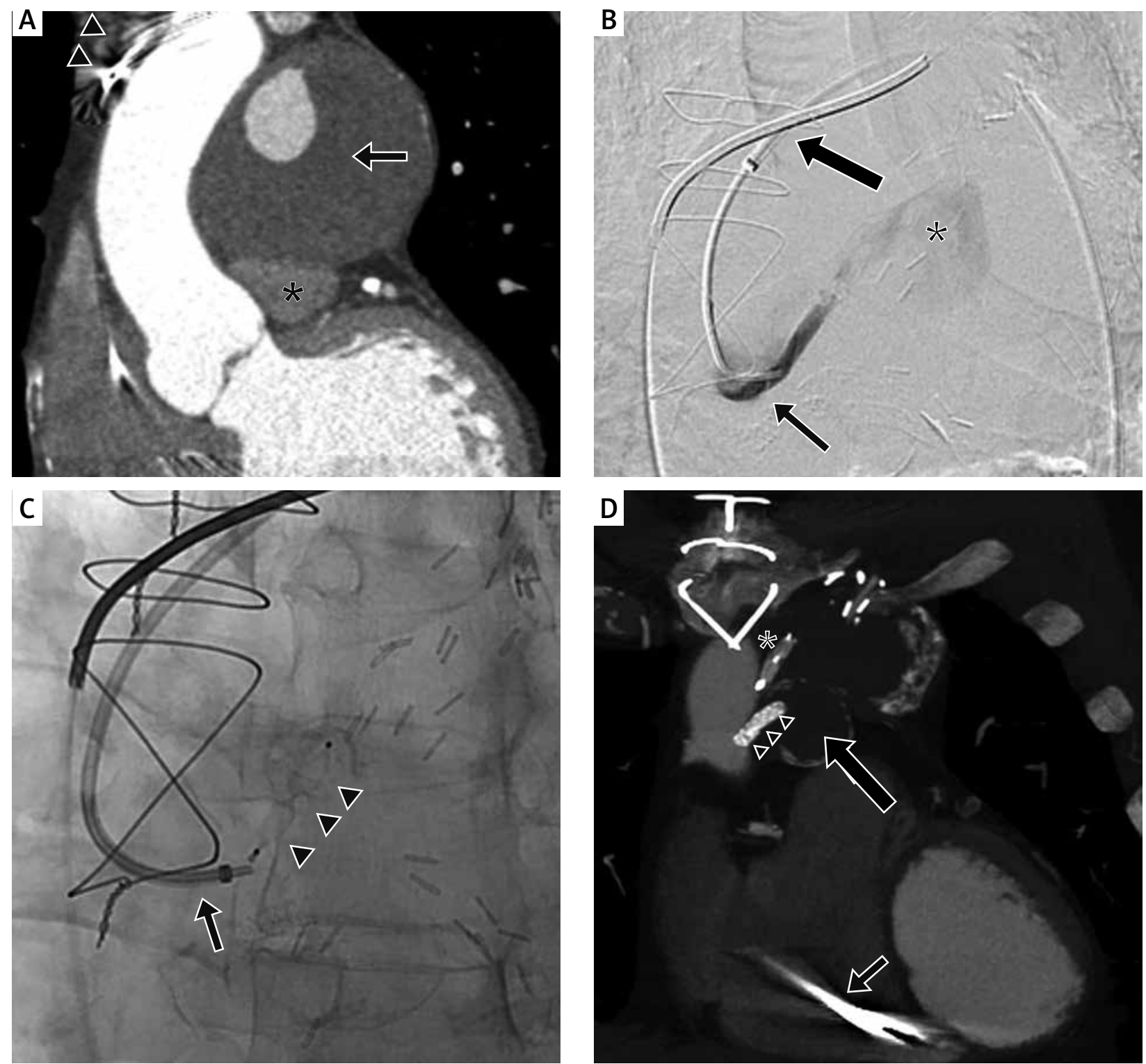

Figure 1. Giant aneurysm of the saphenous vein graft-obtuse marginal branch (SVG-OM) venous bypass graft. A - Coronal reconstruction of the coronary angio-computed tomography (angio-CT) depicting giant, partially thrombosed aneurysm of the SVG-OM bypass graft (arrow) compressing the pulmonary trunk (asterisk). Electrode of the implantable cardioverter-defibrillator directing to the right ventricle visible as well (arrowheads). B - Pre-procedural angiogram of the SVG-OM bypass graft depicting aneurysm filling with contrast medium (asterisk); a $6 \mathrm{Fr} 90 \mathrm{~cm}$-long vascular sheath (Flexor, Cook Medical, IN, USA) was placed at the level of the ascending aorta (thick arrow) through a right femoral access, followed by coaxial, selective positioning of the 5 Fr MPA (Cook Medical, IN, USA) diagnostic catheter in the proximal SVG-OM bypass (arrow). C - Preceded by administration of 3000 IU heparin i.v., a 6 mm Amplatzer Vascular Plug (AVP) 4 (arrowheads) was then deployed with a slight oversizing into the $5 \mathrm{~mm}$ target graft resulting in complete aneurysm exclusion from the circulation; just before AVP deployment the 6 Fr vascular sheath was advanced to the proximal SVG-OM bypass to provide system stability (arrow). The arterial puncture site was closed by StarClose (Abbott Vascular, IL, USA). The patient received low molecular weight heparin $(\mathrm{LMWH})$ S.c. daily for routine postoperative thrombosis prophylaxis, and after discharge was prescribed with optimal medical treatment (acetylsalicylic acid (ASA) 75 mg, statin, angiotensin-converting-enzyme inhibitor (ACEI) and angiotensin II receptor blocker (ARB)) as standard of care, not to impede the effects of AVP with additional antithrombotic agents. D - Control coronary angio-CT at 12 months depicts AVP 4 (asterisk) successfully implanted to the proximal SVG-OM bypass graft, with no residual flow through the aneurysm sac. Fully thrombosed aneurysm of the aorta-diagonal branch-left anterior descending artery (Ao-DIAG-LAD) bypass (thick arrow) with stent deployed within patent graft vessel (arrowheads) is visible as well. Electrode of the implantable cardioverter-defibrillator directing to the right ventricle visible as previously (arrow). Pulmonary trunk decompression is also visible 
nary trunk decompression to $19 \mathrm{~mm}$ in the AP dimension at the 12-month follow-up, and symptom resolution.

\section{Conflict of interest}

The authors declare no conflict of interest.

\section{References}

1. Ramirez FD, Hibbert B, Simard T, et al. Natural history and management of aortocoronary saphenous vein graft aneurysms: a systematic review of published cases. Circulation 2012; 126: 2248-56.

2. Dieter RS, Patel AK, Yandow D, et al. Conservative vs. invasive treatment of aortocoronary saphenous vein graft aneurysms: treatment algorithm based upon a large series. Cardiovasc Surg 2003; 11: 507-13.

3. Weintraub WS, Jones EL, Morris DC, et al. Outcome of reoperative coronary bypass surgery versus coronary angioplasty after previous bypass surgery. Circulation 1997; 95: 868-77. 